\title{
Variability observations of selected southern extragalactic radio sources
}

\author{
G.E. Romero ${ }^{1,2}$, P. Benaglia ${ }^{1,2}$, and J.A. Combi ${ }^{1}$ \\ 1 Instituto Argentino de Radioastronomía, C.C.5, (1894) Villa Elisa, Bs. As., Argentina \\ 2 Now at: Instituto Astronômico e Geofísico, USP, Av. M. Stefano 4200, CEP 04301-904, São Paulo SP, Brazil
}

Received August 27; accepted November 22, 1996

\begin{abstract}
Results of variability observations of the southern extragalactic radio sources PKS 1610 - 771, PKS $1830-211$ and the inner region of Centaurus A (Cen A) at $1.42 \mathrm{GHz}$ are presented. Different light curves with temporal resolutions of $\sim 30$ minutes, 1 day and 1 month were obtained for each source. Significant variability was detected in PKS $1610-771$ and Cen A. The implications of these results are discussed.
\end{abstract}

Key words: galaxies: active - radio continuum: galaxies - galaxies: NGC 5128 - quasars: PKS $1610-$ $771 ;$ PKS $1830-211$

\section{Introduction}

Since 1992 several observational campaigns searching for rapid variability of southern extragalactic radio sources have been carried out at the Instituto Argentino de Radioastronomía (IAR) (Romero et al. 1994, 1995a; Romero \& Combi 1995). These campaigns were conceived with the aim of gathering a set as complete as possible of radio variability data of southern active galactic nuclei (AGNs) on timescales ranging from hours to months at relatively low radio frequencies $(\nu \sim 1.4 \mathrm{GHz})$. Together with the abundant data concerning the behaviour of northern sources (e.g. Quirrenbach et al. 1992) they can provide elements for an all-sky statistics of rapid radio variability of AGNs. In this paper we present results of new observations of the sources PKS 1610 - 771, PKS $1830-211$, and the central region of $\mathrm{Cen} \mathrm{A}$, with different temporal resolutions.

PKS $1610-771$ is a strong $\left(S_{1.4 \mathrm{GHz}} \sim 5 \mathrm{Jy}\right)$, flatspectrum $\left(\alpha_{2.7 \mathrm{GHz}}^{5.0 \mathrm{GHz}} \sim-0.1, S_{\nu} \propto \nu^{\alpha}\right)$ source with a steep optical spectrum (Hunstead \& Murdoch 1980). It has been classified as a QSO by Véron-Cetty \& Véron (1996). The object, with a redshift $z=1.710$, has been observed

Send offprint requests to: G.E. Romero at high angular resolution by the Southern Hemisphere VLBI Experiment (Preston et al. 1989). These observations indicate a $3.8 \mathrm{Jy}$ elliptical Gaussian core elongated 10 mas along a position angle of $35^{\circ}$, and a $1.4 \mathrm{Jy}$ circular Gaussian halo of 25 mas in radius at $2.3 \mathrm{GHz}$. The source, consequently, seems to be compact with no visible structure over the 10 mas. As far as we know there are no reports about radio variability for this object until now.

PKS $1830-211$ is a strong, flat-spectrum source with a double structure at scales of 1 arcsecond. VLBI observations have shown the presence of an Einstein ring and two almost identical bright spots, which have been interpreted as images of a background lensed object (namely a QSO). Owing to its gravitationally lensed nature this system has call much attention in recent years (see, for instance, Jauncey et al. 1991; Nair et al. 1993; van Ommen et al. 1995). The total flux density at $1.4 \mathrm{GHz}$ is $\sim 12 \mathrm{Jy}$ (Pramesh Rao \& Subrahmanyan 1988). Flux density variability has been found at 2.3 and $8.4 \mathrm{GHz}$ by Lovell et al. (1996). At the latter frequency the source evolved from a flux density value of $\sim 5 \mathrm{Jy}$ in late 1990 to a peak of $\sim 11 \mathrm{Jy}$ in early 1992, and then decreasing up to $\sim 7.5 \mathrm{Jy}$. Variability information about this object is important in order to constrain the proposed lens models (e.g. van Ommen et al. 1995).

The third source of our sample, Cen A, is the nearest active galaxy: its distance is $\sim 3.5 \pm 0.2 \mathrm{Mpc}$ (Hui et al. 1993). It is a complex and extremely powerful source in the southern sky, covering an area of about $10^{\circ} \times 5^{\circ}$. The large scale radio structure has been investigated by Cooper et al. (1965), Junkes et al. (1993) and Combi \& Romero (1996), among others. The major radio components of the source are the Northern and Southern Giant Lobes, the Northern Middle Lobe, the Double Inner Lobes, the jet (also visible at X-rays), and the mas-core (see composed maps in Meier et al. 1989). A weak counterjet has been recently discovered with a global (SHEVE + VLBA) array (Jones et al. 1996). The inner radio structure was studied in detail by Burns et al. (1983) with the VLA. Very rapid variability of the central regions of Cen $\mathrm{A}$ at 
mm-wavelengths has been reported by Kellerman (1974) and Kaufmann \& Raffaelli (1979), and variability over larger timescales was detected by Abraham et al. (1982), Botti \& Abraham (1993) and Abraham (1996), among others. Several authors have suggested that Cen A may harbour a misdirected BL Lac object (e.g. Bailey et al. 1986; Morganti et al. 1992). In such a case variability at $\mathrm{cm}$-wavelengths might be produced in the inner region of the source over relatively short timescales (may be months).

In the next section we shall briefly describe our observations of the selected objects. The following sections are devoted to the results and their discussion.

\section{Observations}

The observations were performed in three superposed campaigns in order to obtain three sets of data with different temporal resolutions: $\sim 30$ minutes, 1 day and 1 month. All observations were accomplished with a $30 \mathrm{~m}-$ single dish telescope of the IAR during 1995-1996. This telescope has a coverage from $-9^{\circ}$ to $-90^{\circ}$ in declination and from $-30^{\circ}$ to $+30^{\circ}$ in zenith angle (four hours). The two channel total power receiver with a corrugated horn feed was operated at $1.42 \mathrm{GHz}$ with a bandwidth of $20 \mathrm{MHz}$. At this frequency the HPBW of the antenna is 30 arcminutes. The temperature of the system was $90 \mathrm{~K}$, and the sensitivity in a single record $\sim 30 \mathrm{mK}$. The digitized signal and the telescope position were simultaneously acquired by an IBM computer.

The observing procedure for point sources consisted of repeated orthogonal cross scans in declination and right ascension. The two sets of scans observed for a given source were averaged and Gaussian fitted to determine the peak flux density (which was calibrated using the scale of Wills 1975). A set of standard, steep-spectrum sources was observed for calibration and control purposes: PKS 0915 - 11, PKS 1308 - 22, PKS 1610 - 60, PKS 1814-63, PKS $1932-46$ and PKS 2152-69 (see Table 1). Sources under study are listed in Table 2 together with their coordinates, galactic latitude, redshift and identification.

Cen A is the only extended source in our sample. A different observational technique was used for this object: the observations consisted of scans perpendicular to the line defined by the outer radio galaxy and centered at the nucleus. The length of the scans was 12 HPBW in order to allow a proper determination of the baseline $(8$ HPBW were sufficient for the other sources). Due to the low angular resolution of the telescope we have observed with this procedure not just the nucleus but also the jet and the Inner Lobes of Cen A. Variability, however, must come from the innermost region if the timescales are of the order of a few months. The emission form the outer jet and the Inner Lobes can be considered as a quiescent component $S_{\mathrm{q}}$ in such a way that the total flux density
Table 1. Calibration sources

\begin{tabular}{|c|c|c|c|c|c|c|}
\hline Source & $\begin{array}{c}\alpha_{1950} \\
\left(\begin{array}{c}\mathrm{h} \mathrm{m} \mathrm{s})\end{array}\right.\end{array}$ & $\begin{array}{l}\delta_{1950} \\
\left(0^{\prime} \quad \prime \prime\right)\end{array}$ & $\begin{array}{r}S_{1420} \\
(\mathrm{Jy})\end{array}$ & $\begin{array}{l}\text { Spectral } \\
\text { Index }\end{array}$ & $z$ & Id \\
\hline 0915-11 & 091541.3 & -114305 & 43.8 & -0.88 & 0.052 & G \\
\hline $1308-22$ & 130857.5 & -220042 & 5.40 & -1.24 & $?$ & G \\
\hline $1610-60$ & 161040.8 & -604746 & 54.82 & -1.10 & 0.0176 & G \\
\hline $1814-63$ & 181446.1 & -634701 & 13.38 & -0.75 & 0.063 & G \\
\hline $1932-46$ & 193218.9 & -462724 & 13.07 & -1.06 & $?$ & G \\
\hline 2152-69 & 215301.6 & -695546 & 30.40 & -0.81 & 0.027 & G \\
\hline
\end{tabular}

G: Radio galaxy.

Table 2. Target sources

\begin{tabular}{lcccccc}
\hline Source & $\begin{array}{c}\alpha_{1950} \\
(\mathrm{~h} \mathrm{~m} \mathrm{~s})\end{array}$ & $\begin{array}{c}\delta_{1950} \\
\left({ }^{\prime}{ }^{\prime \prime}\right)\end{array}$ & $\begin{array}{c}b \\
\left({ }^{\circ}\right)\end{array}$ & $z$ & Id \\
\hline Cen A & 132236.0 & -4245 & 45 & +19.4 & 0.002 & G \\
$1610-771$ & 161051.7 & -770952 & -18.9 & 1.710 & Q \\
$1830-211$ & 183040.6 & -210600 & -5.7 & $?$ & Q? \\
\hline
\end{tabular}

Q: QSO, G: Radio galaxy.

observed is $S_{\text {obs }}(t)=S_{\mathrm{q}}+S_{\mathrm{v}}(t)$, where $S_{\mathrm{v}}(t)$ is the variable component.

The errors quoted with the variability data presented in this paper are rms fluctuations of the flux densities obtained from each set of scans. These errors are contributed by $(i)$ absolute errors (i.e. errors with no dependence on the flux density of the observed sources), and (ii) intensity-proportional errors. Errors of type $(i)$ are due to the noise and short-term instabilities of the receiver, confusion, and low-level interference. Errors of type (ii) are mainly originated in antenna pointing errors and variations of gain with zenith angle. In case of daytime observations there are additional contributions to the total errors from solar activity and differential heating and cooling of the antenna.

In order to determine the error budget we have studied the scatter of the peak flux densities associated to scans obtained within individual source observations. The rms fluctuations have been plotted against the flux densities of a large number of sources of different intensities. Errors of type (ii) are dominant for large flux densities whereas errors of type $(i)$ are important for relatively weak sources. In both domains the errors can be fitted by linear 
functions, allowing a numerical determination. The total rms error for a flux density value $S$ is then given by:

$\epsilon_{S}=\sqrt{\epsilon_{\mathrm{a}}^{2}+\epsilon_{\mathrm{p}}^{2} S^{2}}$,

where $\epsilon_{\mathrm{a}} \approx 0.18 \pm 0.01 \mathrm{Jy}$ and $\epsilon_{\mathrm{p}} \approx 2 \%$. For daytime observations the absolute errors are larger: $\epsilon_{\mathrm{a}} \approx 0.21 \pm$ $0.01 \mathrm{Jy}$. The confusion contribution to $\epsilon_{\mathrm{a}}$ is $0.08 \mathrm{Jy}$. In the case of the weakest sources of our sample (PKS 1610-771) the total errors are about $4 \%$ of the flux density.

In addition to these errors for individual observations, variations on the gain and sensitivity of the entire observing system can introduce a spurious low-level variability in the observed light curves. This effect can be determined by observations of calibration sources as we shall describe in the next section.

\section{Results}

Results of the observations are presented in Figs. 1-3 as residual light curves (numerical values are available upon request in the form of an ASCII file). Residuals are defined as:

$R_{i}=\frac{100\left(S_{i}-<S>\right)}{<S>}$

where $\langle S\rangle$ is the mean flux density. Figure 1 shows light curves with temporal resolution of $\sim 30$ minutes. Figures 2 and 3 contain residuals with resolution of 1 day and 1 month, respectively. Residual curves corresponding to steep-spectrum sources have been included in the figures for comparison.

In order to check the presence of variability in the different light curves a $\chi^{2}$-test with a confidence level of $99.9 \%$ was applied. No variability within the measurement errors was detected at intraday resolution. Interday variability was present in PKS 1610-771. Cen A seems to be non-variable over timescales of weeks or less at $1.4 \mathrm{GHz}$. However, variations of $\sim 10 \%$ were detected over timescales of months. Two bursts can be observed in the light curve shown in Fig. 3. PKS 1610 - 771 was also variable over large timescales.

The observed variability can be characterized by a percentage fluctuation index:

$\mu=100 \frac{\sigma_{S}}{<S>}$.

Fluctuations of the steep-spectrum sources included in the sample for control purposes were then interpreted as spurious variability introduced by the observing system. If $\mu_{0}$ is the largest fluctuation index of calibration sources during a campaign with temporal resolution $\Delta t$, then the real variability of the source under study can be measured by an amplitude $Y_{\Delta t}$ given by (e.g. Quirrenbach et al. 1992):

$Y_{\Delta t}=3 \sqrt{\mu^{2}-\mu_{0}^{2}}$.
Variability parameters for the three sources of our sample are given in Table 3: source name, number of points in the light curve, mean flux density, result of the $\chi^{2}$-test (V: variable, NV: non variable), fluctuation index, variability amplitudes, associated timescales, activity parameter $\dot{S}$, and the slope of the corresponding structure function (see below), from left to right. Typical values of $\mu_{0}$ are $\sim 2 \%$, except for the intraday observations of PKS $1610-771$ where larger values were observed $(\sim 3.1 \%)$.

Table 3. Variability parameters

\begin{tabular}{|c|c|c|c|c|c|c|c|c|}
\hline Source & $n$ & $<\underset{(\mathrm{Jy})}{S>}$ & $\chi^{2}$ & $\begin{array}{c}\mu \\
(\%)\end{array}$ & $\begin{array}{l}Y \\
(\%)\end{array}$ & $\begin{array}{c}\tau \\
\text { (days) }\end{array}$ & $\begin{array}{c}\dot{S} \\
(\mathrm{Jy} / \text { day })\end{array}$ & $\alpha$ \\
\hline \multicolumn{9}{|l|}{ PKS 1610-771 } \\
\hline intraday & 11 & 4.65 & $\mathrm{NV}$ & 3.5 & - & - & - & - \\
\hline interday & 15 & 5.00 & $\mathrm{~V}$ & 7.1 & 19.3 & 3.5 & 0.44 & 1.1 \\
\hline intermonth & 15 & 4.64 & $\mathrm{~V}$ & 7.6 & 21.1 & $\sim 180$ & 0.006 & 1.2 \\
\hline \multicolumn{9}{|l|}{ PKS 1830-211 } \\
\hline intraday & 11 & 13.57 & $\mathrm{NV}$ & 1.1 & - & - & - & - \\
\hline interday & 14 & 13.77 & $\mathrm{NV}$ & 3.1 & 6.8 & - & - & - \\
\hline intermonth & 11 & 13.43 & $\mathrm{NV}$ & 2.6 & 4.6 & - & - & - \\
\hline \multicolumn{9}{|l|}{ Cen A } \\
\hline intraday & 7 & 299.68 & $\mathrm{NV}$ & 0.4 & - & - & - & - \\
\hline interday & 15 & 299.37 & NV & 0.6 & - & - & - & - \\
\hline intermonth & 14 & 292.18 & $\mathrm{~V}$ & 4.3 & 9.4 & $\sim 100$ & 1.23 & 1.1 \\
\hline
\end{tabular}

The timescales in Table 3 were determined by means of the first-order structure functions introduced by Simonetti et al. (1985):

$D^{I}(T)=<(R(t)-R(t+T))^{2}>$,

where $R(t)$ is the residual at time $t$ and the average is taken over all pairs of observations with time lag $T$. The maxima in the $\log D^{I}(T)-\log T$ plane characterize the timescales of a given source. The slope $\alpha$ of the structure functions can be used to investigate the nature of the underlying physical process (e.g. Qian et al. 1995). Structure functions for the light curves with variability are shown in Fig. 4. The structure function of PKS $1830-211$ at interday resolution is also shown in this figure.

PKS 1830-211 has been classified as NV due to its flat structure function and the result of the $\chi^{2}$-test. However, a small variability amplitude can be assigned to the interday and intermonth light curves owing to a one-point deviation in each curve (see Figs. 2, 3 and 4). These amplitudes have been included in Table 2 . The possibility of a real variation with a timescale of $\sim 1$ day cannot be ruled out.

\section{Discussion}

We have not detected short-term variability in Cen A. The detection of percentage variations of extremely bright 

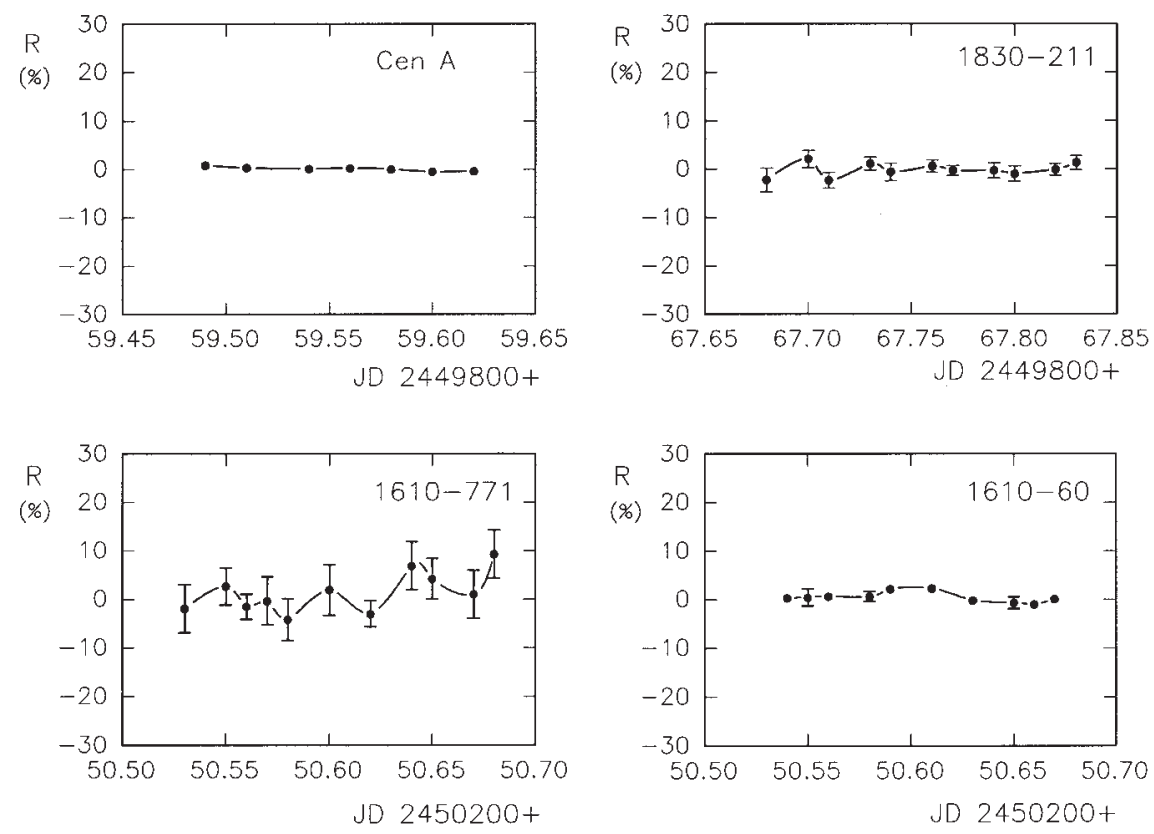

Fig. 1. Residual light curves for intraday observations
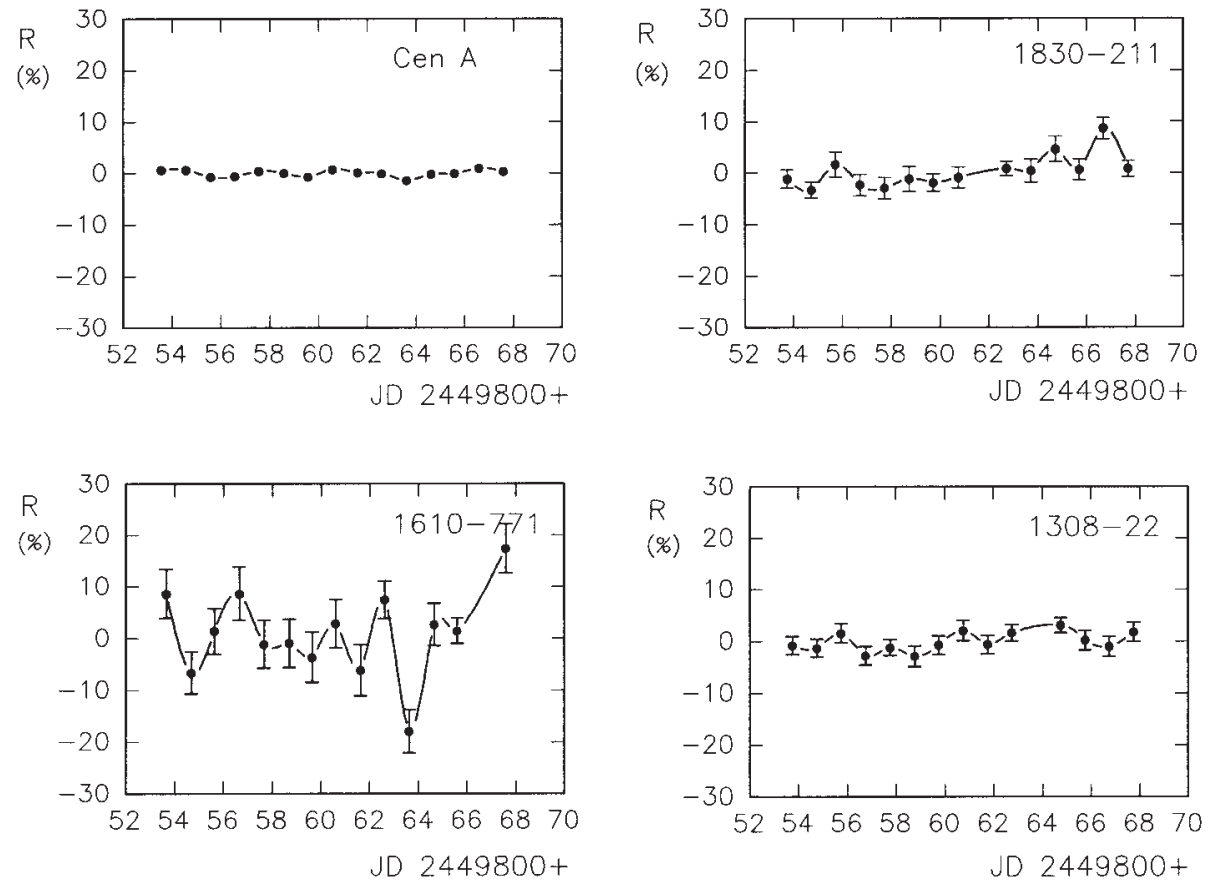

Fig. 2. Residual light curves for interday observations 

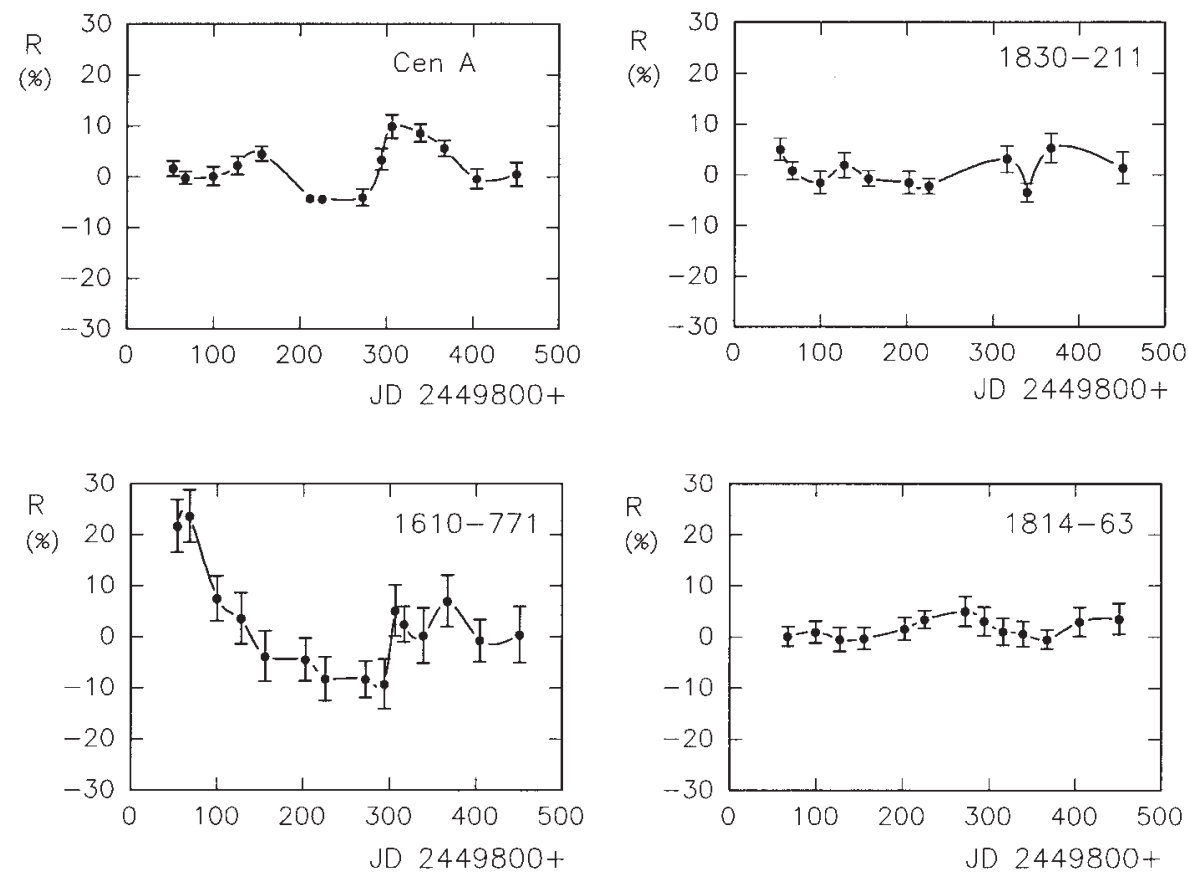

Fig. 3. Residual light curves for intermonth observations
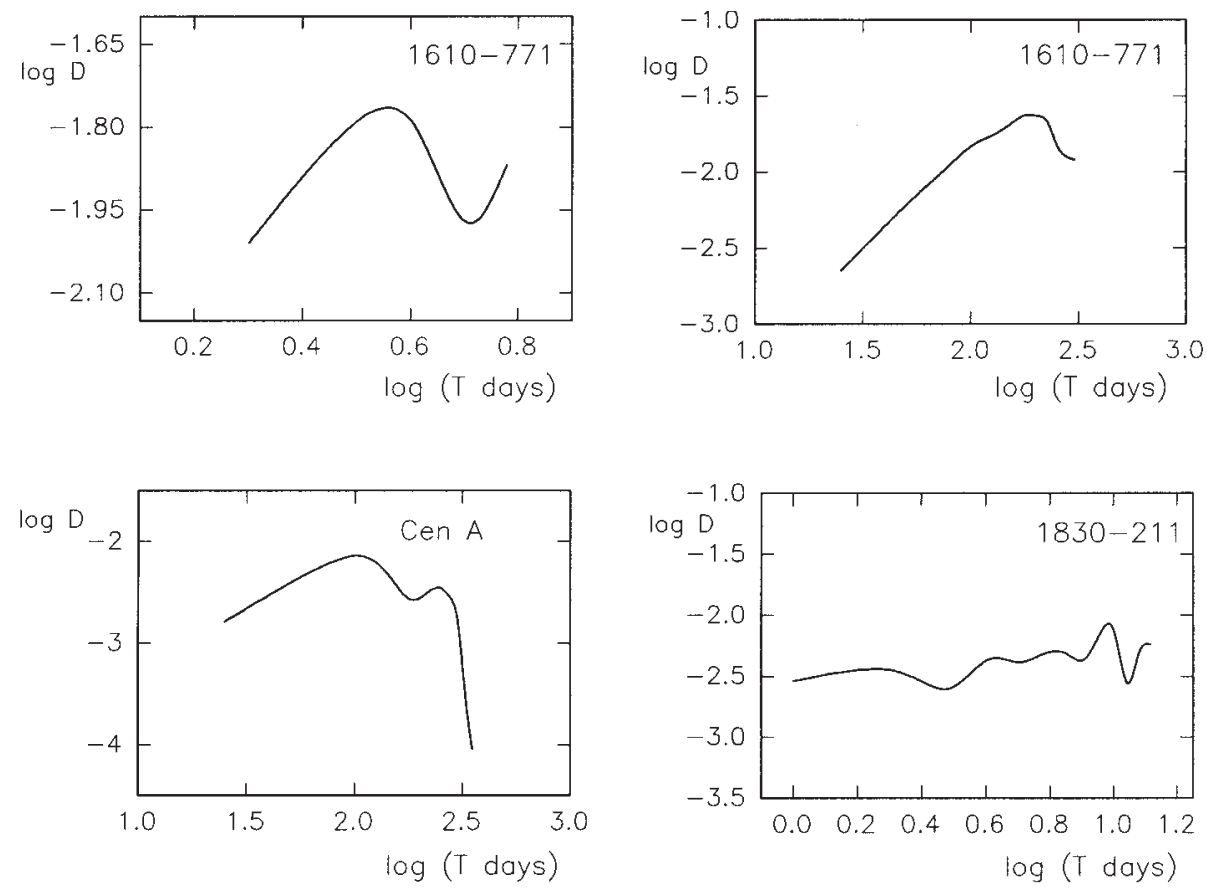

Fig. 4. First-order structure functions 
sources can be limited by the dynamic range of the receiver. However, in the case of the present observations we have a range of $\sim 20 \mathrm{~dB}$ which allows us a proper determination of fluctuations on the percent level for sources with flux densities even beyond $10^{3} \mathrm{Jy}$. Consequently, we conclude that significant flux density variations in Cen A seem to be confined to timescales larger than 1 month at $1.4 \mathrm{GHz}$. At larger frequencies, however, there are reports of strong rapid changes in flux density (for instance, Kellerman 1974 has observed 50\% fluctuations over one day at $88 \mathrm{GHz}$ ). Our data (see Fig. 3) show variability with amplitudes $Y \sim 9.4 \%$ over timescales of $\sim 100$ days. The intensity parameter $\dot{S}$ is about $1.23 \mathrm{Jy} /$ day, implying an upper limit for the linear size of the emitting region of $\sim 0.5 \mathrm{pc}$. The corresponding brightness temperature for the synchrotron plasma that produces this emission $(\Delta S \sim 33 \mathrm{Jy})$ is $\sim 2.710^{10} \mathrm{~K}$, a value quite smaller than the inverse Compton limit. There is no need, consequently, for positing relativistic bulk motions in the source in order to reduce the derived brightness temperature in the observer's frame. This is in accordance with recent VLBI observations of Cen A which show subluminal velocities of $\sim 0.15 \mathrm{c}$ in the inner jet of the object (Preston et al. 1996). The observed variability might be produced when a shock strikes a small feature (e.g. a density inhomogeneity) in the pc-scale jet (Romero et al. 1995c). This interpretation is supported by the knotty structure of the inner radio jet observed, for instance, by Burns et al. (1983). Besides, there seems to be unlikely that the variations could be originated in the core alone due to its relatively low contribution to the total flux density at $1.4 \mathrm{GHz}$.

With the exception of small fluctuations between JD 2449866 and JD 2449868, and JD 2450016 and JD 2450030, we have observed no variability in the gravitational lensed system PKS 1830 - 211. Gravitational microlensing might cause variability in this source: compact objects like stars or brown dwarfs belonging to the lensgalaxy can magnify the emission from the nucleus or a superluminal component in the jet of the background AGN producing rapid and symmetric changes in the flux density (e.g. Nottale 1986; Gopal-Krishna \& Subrahmanian 1991). Variations over timescales of days require extremely high velocities of the lens with respect to the observer or, more reasonably, a superluminal lensed source. Our fail in clearly detecting these variations suggests that superluminal components with a significant part of the total flux density were not present in the source during the observational period. Future variability observations with better sensitivity of PKS 1830 - 211 could provide a valuable tool for investigating the nature and structure of the background source and the interposed galaxy, assuming the corresponding redshifts can be obtained (see Romero et al. 1995b for a treatment of this kind).

The QSO $1610-771$ presented the larger variability amplitudes of the sample. At intraday timescales there is no variability over the observational errors, but at timescales of months variability amplitudes of $\sim 21 \%$ were observed. Superposed with this variability there are rapid fluctuations of a few days. The fastest changes in flux density have a peak-to-peak amplitude of $\Delta R \sim 40 \%$ in 4 days. The intensity parameter $\dot{S}$ is of $\sim 0.44 \mathrm{Jy} /$ day for these variations. This kind of events implies large brightness temperatures well beyond the inverse Compton limit $\left(T_{\mathrm{B}} \sim 7.210^{17} \mathrm{~K}\right.$, for $q_{0}=1 / 2$ and $H_{0}=$ $100 \mathrm{~km} \mathrm{~s}^{-1} \mathrm{Mpc}^{-1}$ ) if they are interpreted as intrinsic to the source. Shocked jet models with favorable geometries (e.g. Qian et al. 1991; Romero et al. 1995c) require bulk Lorentz factors as high as 15 to account for these observations. Conversely, the temperatures derived from the intermonth variability data are easily reconcilable with the standard shock-in-jet model of blazars (e.g. Marscher 1992). One possibility to be considered is that the fast variations are produced by refractive interstellar scintillation (Rickett 1986) whilst the variation over larger timescales could have an intrinsic origin. This hypothesis is supported by the fact that the interday structure function is linear in $T$ for $T$ small, in agreement with the theoretical predictions for the variability produced by an extended scattering medium (Blandford et al. 1986). The observed fluctuation index implies, in this interpretation, that a significant part of the flux density is within a $\sim 0.1$ mas core.

Beyond the origin of the short-term variability of PKS $1610-771$, it is clear that this object should receive more attention in the future.

Acknowledgements. We thank E. Hurrell for assistance during the observations, and A. Bava and J. Sánz for technical advise. This work has been partially supported by CONICET and UNLP.

\section{References}

Abraham Z., Kaufmann P., Botti L.C.L., 1982, AJ 87, 532

Abraham Z., 1996, in Extragalactic Radio Sources, Fanti C. (ed.). Kluwer, Dordrecht, p. 25

Bailey J., Sparks W.B., Hougn J.H., Axon D.S., 1986, Nat 322, 150

Blandford R., Narayan R., Romani R.W., 1986, ApJ 301, L53

Botti L.C.L., Abraham Z., 1993, MNRAS 264, 807

Burns J.O., Feigelson E.B., Schreier E.J., 1983, ApJ 273, 128

Combi J.A., Romero G.E., 1997, A\&AS 121, 11

Cooper B.F.C., Price R.M., Cole D.J., 1965, Austr. J. Phys. 18,589

Gopal-Krishna, Subrahmanian K., 1991, Nat 349, 766

Hui X., Fura H.C., Ciaraullo R., Jacury G.H., 1993, ApJ 414, 463

Hunstead R.W., Murdoch H.S., 1980, MNRAS 192, 31P

Jauncey D.L., Reynolds J.E., Tzioumis A.K., et al., 1991, Nat 352,132

Jones D.L., Tingay S.J., Preston R.A., et al., 1996, in Extragalactic Radio Sources, Fanti C. (ed.). Kluwer, Dordrecht, p. 23

Junkes N., Haynes R.F., Harnett J.I., Jauncey D.L., 1993, A\&A 269, 29

Kaufmann P., Rafaelli J.C., 1979, MNRAS 187, 23P 
Kellerman K.I., 1974, ApJ 194, L135

Lovell J.E.J., Mcculloch P.M., King E.A., Jauncey D.L., 1996, in Astrophysical Applications of Gravitational Lensing, Kochanek C.S. and Hewitt J.N. (eds.). Kluwer, Dordrecht, p. 347

Marscher A.P., 1992, in Physics of Active Galactic Nuclei, Duschl W.J. and Wagner S.J. (eds.). Springer-Verlag, Heidelberg, p. 510

Meier D.L., Jauncey D.L., Preston R.A., 1989, AJ 98, 27

Morganti R., Fosbury R.A.E., Hock R.N., et al., 1992, MNRAS 256, 1P

Nair S., Narasimha D., Pramesh Rao A., 1993, ApJ 407, 46

Nottale L., 1986, A\&A 157, 383

van Ommen T.D., Jones D.L., Preston R.A., Jauncey D.L., 1995, ApJ 444, 561

Pramesh Rao A., Subrahmanyan R., 1988, MNRAS 231, 229

Preston R.A., Jauncey D.L., Meier D.L., et al., 1989, AJ 98, 1

Preston R.A., Tingay S.J., Jauncey D.L., et al., 1996, in Extragalactic Radio Sources, Fanti C. (ed.). Kluwer, Dordrecht, p. 21

Qian S.J., Quirrenbach A., Witzel A., et al., 1991, A\&A 241, 15

Qian S.J., Britzen S., Witzel A., et al., 1995, A\&A 295, 47

Quirrenbach A., Witzel A., Krichbaum T., et al., 1992, A\&A 258,279

Rickett B.J., 1986, ApJ 307, 564

Romero G.E., Combi J.A., Colomb F.R., 1994, A\&A 288, 731

Romero G.E., Combi J.A., 1995, Ap\&SS 229, 23

Romero G.E., Benaglia P., Combi J.A., 1995a, A\&A 301, 33

Romero G.E., Surpi G., Vucetich H., 1995b, A\&A 301, 641

Romero G.E., Combi J.A., Vucetich H., 1995c, Ap\&SS 225, 183

Simonetti J.H., Cordes J.M., Heeschen D.S., 1985, ApJ 296, 46

Véron-Cetty M.P., Véron P., 1996, ESO Scientific Report No. 17

Wills B.J., 1975, Aust. J. Phys. Astrophys. Suppl. 28, 1 